\title{
Compact Fully Uniplanar Bandstop Filter Based on Slow-Wave Multimodal CPW Resonators
}

\author{
Adrián Contreras, Miquel Ribó, Member, IEEE, Lluís Pradell ${ }^{\circledR}$, Member, IEEE, Victor Raynal, \\ Ignacio Moreno, Maxence Combes, and Milena Ten
}

\begin{abstract}
In this letter, a compact, fully uniplanar bandstop filter is presented. It is based on multimodal coplanar waveguide resonators implemented using a slow-wave periodic structure that greatly reduces the filter's horizontal dimension. A multimodal circuit model for the filter is proposed and experimentally validated. A second-order filter prototype at $1.9 \mathrm{GHz}$, with a compact size of $0.31 \lambda_{g} \times 0.19 \lambda_{g}$, was designed and fabricated. The filter features measured fractional bandwidths of $9.3 \%$ and $3.7 \%$ for -20 - and $-30-\mathrm{dB}$ rejection levels, respectively, and a 1.3-dB insertion loss in the passband.
\end{abstract}

Index Terms-Bandstop filter (BSF), multimodal circuit, slow wave, uniplanar.

\section{INTRODUCTION}

$\mathbf{U}$ NIPLANAR transmission lines such as the coplanar waveguide (CPW), the coplanar stripline, and the slotline offer various advantages with respect to microstrip and other multilayer structures, due to their simpler fabrication process not requiring via holes (which can be unfeasible in specific substrates [1]) and the associated cost reduction. Fully uniplanar CPW bandstop filters (BSFs) using symmetric slotline stubs [2], split-ring resonators [3], open-loop resonators [4], or coupled-line sections [1] have been reported in the literature. Slotted or patterned-ground resonators have also been used to design BSFs but, in contrast to the former, their implementation is not fully uniplanar since the resonators are vertically coupled to microstrip lines [5]-[8], or a multilayer structure is used for biasing purposes [9].

The CPW is a multimodal waveguide able to simultaneously propagate two fundamental modes (even and odd) which interact at any asymmetry or transition [10], [11]. In filter designs, the odd mode is usually considered spurious and it is suppressed by using air bridges [1], or it does not propagate because of the circuit symmetry [2], [3], [8], [9]. However,

Manuscript received November 28, 2017; accepted July 9, 2018. This work was supported in part by the Spanish Ministerio de Economia y Competitividad under Project TEC2013-48102-C2-1/2-P, Project TEC201678028-C3-1-P, and Grant BES-2008-004923, and in part by the Agencia Estatal de Investigación, Spain, through the Unidad de Excelencia Maria de Maeztu under Grant MDM-2016-0600. (Corresponding author: Lluís Pradell.)

A. Contreras, L. Pradell, V. Raynal, I. Moreno, M. Combes, and M. Ten are with the Department of Signal Theory and Communications, Universitat Politècnica de Catalunya, 08034 Barcelona, Spain (e-mail: pradell@ tsc.upc.edu).

M. Ribó is with the Electronics and Telecommunications Department, Ramon Llull University, La Salle Campus, 08022 Barcelona, Spain (e-mail: mrp@salleurl.edu).

Color versions of one or more of the figures in this paper are available online at http://ieeexplore.iee.org.

Digital Object Identifier 10.1109/LMWC.2018.2855565

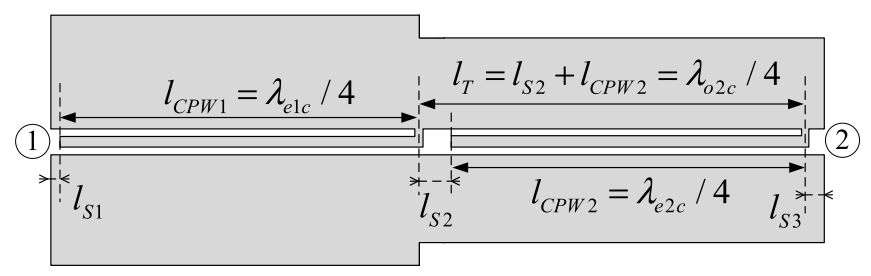

Fig. 1. Uniplanar CPW second-order BSF.

the CPW multimodal behavior offers a further degree of freedom in the design of uniplanar filters which has already been exploited in bandpass designs to obtain harmonic band suppression or a compact size [10]. However, its possibilities for the BSF design have not been explored yet.

In this letter, a compact, fully uniplanar multimodal BSF is presented. It is based on multimodal CPW resonators, which combine in the same area a resonator and a part of an immittance inverter. The resonator length is reduced using a CPW slow-wave periodic structure. A multimodal circuit model and a systematic design procedure for the filter are proposed. To demonstrate the filter concept, a second-order prototype with a center frequency of $1.9 \mathrm{GHz}$ was fabricated. The multimodal circuit model and the design procedure are validated by comparing simulation results and measurements.

\section{Filter Structure And Design}

\section{A. Bandstop Filter Structure}

The proposed filter is shown in Fig. 1. It is composed of two multimodal CPW resonators (of length $l_{\mathrm{CPW} i}, i=1,2$ ) embedded in slotline sections (in this letter, subscripts $e, o$, $S, 1,2$, and $c$ refer to the even, odd, and slotline modes, the first and second resonators, and the filter center frequency, respectively). The even and odd modes coexist in the CPW sections. At the two slotline-to-CPW symmetric transitions (on the left side of the resonators), the slotline mode is converted into the CPW odd mode, and vice versa [10], due to their modal compatibility (both modes also feature very similar characteristic impedances and phase velocities, $Z_{0 \mathrm{oi}} \cong Z_{0 \mathrm{Si}}$ and $v_{\text {poi }} \cong v_{\mathrm{pSi}}, i=1,2$ ). The even mode is also generated at the two CPW-to-slotline asymmetric transitions featuring a shunt short circuit in the upper CPW slot (on the right side of the resonators) [11]. From there, it can only propagate backward along the CPW section until it reaches an open circuit at the other end of the CPW section. Using the multimodal circuit model for asymmetric shunt impedances [11], the circuit of Fig. 1 can be rigorously modeled as shown in Fig. 2 . 


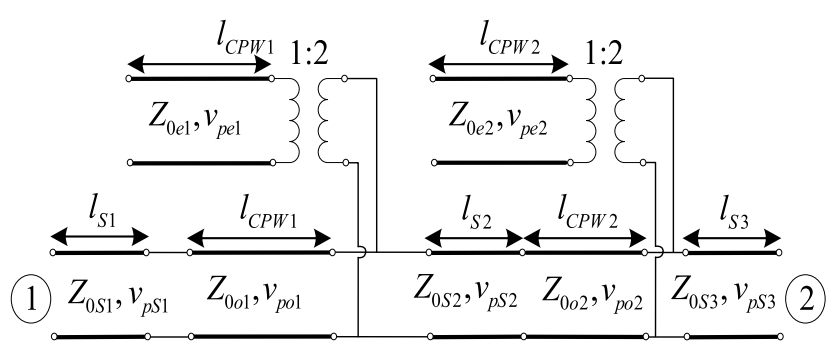

Fig. 2. Multimodal equivalent circuit of the BSF in Fig. 1.

The even-mode sections are shunt connected to the slotline and CPW-odd-mode cascaded sections, generating a structure well suited to implement BSFs. Indeed, the slotline-CPW-oddmode section of length $l_{T}=l_{S 2}+l_{\mathrm{CPW} 2}=\lambda_{o 2 c} / 4$ acts as an immittance inverter and the shunt CPW-even-mode openended stubs of length $l_{\mathrm{CPW} 1}=\lambda_{e 1 c} / 4$ and $l_{\mathrm{CPW} 2}=\lambda_{e 2 c} / 4$ as quarter-wavelength resonators. Therefore, the CPW-evenmode shunt resonators are embedded in the slotline-CPW-oddmode inverter sections, thus generating potentially compact uniplanar structures since different circuit functions share the same circuit area. To guarantee $l_{T}>l_{S 2}$, the even- and oddmode wavelengths of the second CPW section should fulfill $\lambda_{e 2 c}<\lambda_{o 2 c}$. This condition implies different even- and oddphase velocities and can more appropriately be controlled using a slow-wave structure as described in Section II-B.

The characteristic impedances of the various CPW and slotline sections are calculated as a function of the fractional bandwidth (FBW) and the normalization impedance $Z_{0}(=$ $\left.Z_{0 S 1}=Z_{0 o 1}\right)$ using [12]

$$
\begin{aligned}
& Z_{0 e 1}=\left(Z_{0} / 4\right)+\left(Z_{0} /\left(4 \alpha g_{0} g_{1}\right)\right) \\
& Z_{0 e 2}=\left(Z_{0} g_{0}\right) /\left(4 \alpha g_{2}\right) \\
& Z_{0 o 2}=Z_{0 S 2}=Z_{0} \cdot\left(1+\left(\alpha g_{0} g_{1}\right)\right) \\
& Z_{0 S 3}=Z_{0} g_{0} g_{3}
\end{aligned}
$$

where $\alpha=\cot [(\pi / 2) \cdot(1-(\mathrm{FBW} / 2))]$ and $g_{i}(i=0, \ldots, 3)$ are the normalized low-pass prototype element values.

\section{B. Filter Implementation Using Slow-Wave Periodic Structures}

To reduce the filter's horizontal dimension, the straight CPWs are replaced with the CPW slow-wave periodic structure shown in Fig. 3. It consists of a CPW section with equally spaced ( $p$ dimension) vertical, symmetric CPW stubs of length $l_{B}$. The CPW stubs are open ended for the CPW even mode and short ended (at a distance $h_{1}$ from the metallization edge) for the $\mathrm{CPW}$ odd mode. By removing the central conductor, this structure becomes a slow-wave slotline with short-ended stubs. The periodic stubs increase the even-mode capacitance and the odd-mode (and slotline mode) inductance per unit length. Therefore, the phase velocities decrease (slow-wave effect), $Z_{0 e}$ decreases, and $Z_{0 o}$ and $Z_{0 S}$ increase. Table I compares the calculated phase velocities and mode impedances using 2.5-D electromagnetic simulation for the slow-wave structure of Fig. 3 on an alumina substrate $\left[\varepsilon_{r}=9.9, \tan (\delta)=0.0002\right.$, and thickness $\left.=1016 \mu \mathrm{m}\right]$, and gold metallization (thickness $=4 \mu \mathrm{m}$ ), with fixed total
TABLE I

Simulated Normalized PHASE Velocities ANd IMPEDANCES For the Slow-WAVE Structure of FIg. $3(f=1.9 \mathrm{GHz})$

\begin{tabular}{ccccccc}
\hline \hline$p(\mu \mathrm{m})$ & $l_{B}(\mu \mathrm{m})$ & $h_{1}(\mu \mathrm{m})$ & $v_{p e}{ }^{*}$ & $Z_{0 e}(\Omega)$ & $v_{p o}{ }^{*}$ & $Z_{0 o}(\Omega)$ \\
\hline 700 & 925 & 2755 & 0.40 & 44 & 0.51 & 69 \\
700 & 925 & 100 & 0.31 & 54 & 0.31 & 114 \\
700 & 3580 & 100 & 0.18 & 32 & 0.22 & 77 \\
1400 & 3580 & 100 & 0.28 & 37 & 0.31 & 78 \\
straight & 0 & 4030 & 1 & 64 & 0.92 & 53 \\
CPW & & & & & & \\
\hline \hline
\end{tabular}

${ }^{*}$ The mode phase velocities are normalized to $v_{p e}$ of a straight $\mathrm{CPW}$

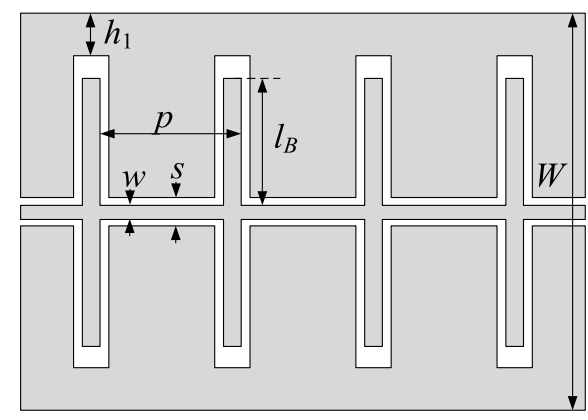

Fig. 3. Uniplanar slow-wave CPW structure.

width $(W=8160 \mu \mathrm{m})$, slot width $(s=100 \mu \mathrm{m})$, strip width $(w=50 \mu \mathrm{m})$, and different values of $l_{B}, p$, and $h_{1}$. It is observed that the smaller $p$ (the more CPW stubs in a fixed-length section) and the longer $l_{B}$, the smaller the phase velocities. Moreover, the condition $\lambda_{p e 2 c}<\lambda_{p o 2 c}$ required for physical implementation of the filter of Fig. 1 is fulfilled. The odd-mode impedance $Z_{0 o}$ is very sensitive to $h_{1}$. The smaller $h_{1}$, the larger is $Z_{0 o}$ for a fixed $l_{B}$. Using $p=700 \mu \mathrm{m}$, the phase velocity decreases up to $82 \%$ (even mode) and $77 \%$ (odd mode). Since the even and odd modes coexist in the CPW sections, $h_{1}, l_{B}$, and $p$ must be chosen to meet (1) and to reduce the phase velocity (for filter-length reduction) according to Table I.

\section{EXPERIMENTAL RESUlts}

A Butterworth BSF with center frequency $f_{0}=1.9 \mathrm{GHz}$ and $-3-\mathrm{dB} \mathrm{FBW}=25 \%$ was fabricated on the alumina substrate (Fig. 4). Its characteristic impedances, computed using (1) with $Z_{0}=Z_{0 S 1}=50 \Omega$, are $Z_{0 e 1}=56.9 \Omega$, $Z_{0 e 2}=44.4 \Omega, Z_{0 o 2}=64 \Omega$, and $Z_{0 S 3}=50 \Omega$. The normalized phase velocities are $0.65(0.4)$ for the even mode of the first (second) slow-wave resonator and 0.51 for the odd mode of the second slow-wave CPW section. The filter size is $20.2 \mathrm{~mm} \times 12.6 \mathrm{~mm}$ or $0.31 \lambda_{\mathrm{gc}} \times 0.19 \lambda_{\mathrm{gc}}$, where $\lambda_{\mathrm{gc}}$ is the wavelength of a straight-CPW even mode. Fig. 5 compares the simulated and measured filter stopband performances, showing an excellent agreement, thus validating the multimodal model proposed in Fig. 2. The simulation uses the equivalent circuit of Fig. 2 and takes into account transmission line losses and small parasitic effects (open-ended capacitance of the CPW even-mode stubs and series inductance due to the change in the metallization widths). The simulated and measured FBW 


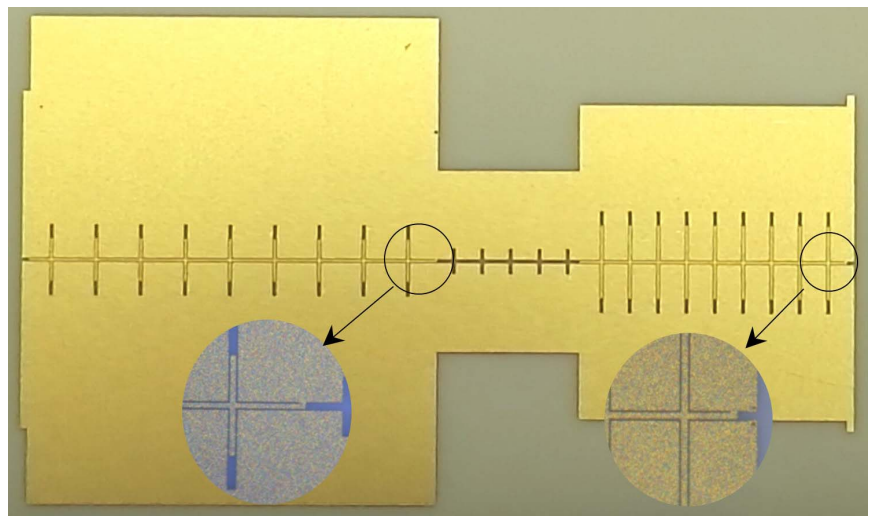

Fig. 4. Uniplanar slow-wave BSF fabricated on an alumina substrate Dimensions according to Fig. 3 (in $\mu \mathrm{m}$ ): $l_{B}=525 / 925, p=1100 / 700$, and $h_{1}=5400 / 2755$ (first/second resonator). $p=700$ and $h_{1}=2015$ (central slotline).

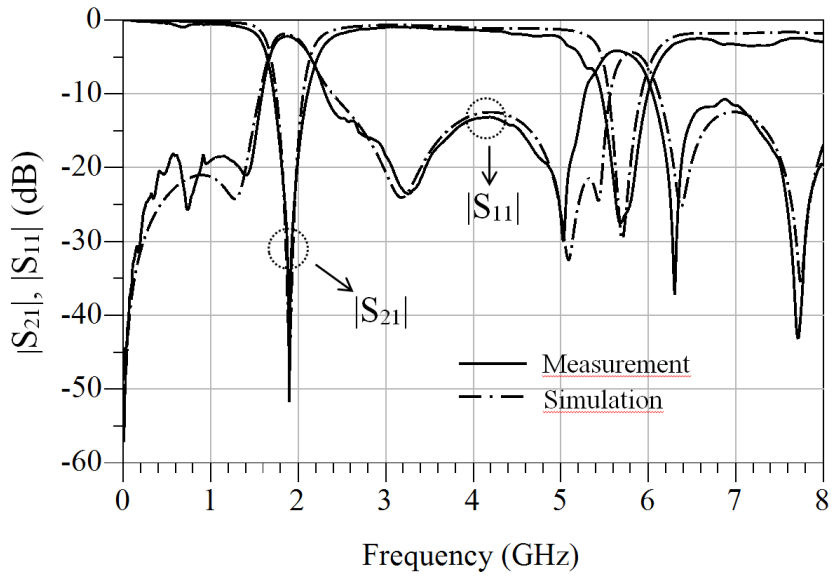

Fig. 5. Simulated and measured BSF performance.

TABLE II

COMPARISON WITH OTHER FULLY UNIPLANAR AND NOT-UNIPLANAR BSFS

\begin{tabular}{ccccccc}
\hline \hline & $\begin{array}{c}f_{0} \\
(\mathrm{GHz})\end{array}$ & $\begin{array}{c}\text { Filter } \\
\text { order } \\
(n)\end{array}$ & $\begin{array}{c}-20-\mathrm{dB} \\
\text { FBW }\end{array}$ & $\begin{array}{c}-30-\mathrm{dB} \\
\text { FBW }\end{array}$ & $\begin{array}{c}\text { Passband } \\
\mathrm{IL}, f \leq 2 f_{0}\end{array}$ & Size \\
\hline$[2]$ & 4.9 & 3 & $22 \%{ }^{*}$ & $7.5 \%^{*}$ & $2 \mathrm{~dB}^{*}$ & $0.6 \lambda_{g c} \times 0.5 \lambda_{g c}{ }^{*}{ }^{*}$ \\
{$[3]$} & 7 & 3 & $1.2 \%^{*}$ & - & $1 \mathrm{~dB}^{*}$ & $0.64 \lambda_{g c} \times 0.3 \lambda_{g c}{ }^{*}{ }^{*}{ }^{*}$ \\
{$[4]$} & 3.4 & 2 & $20 \%{ }^{*}$ & $2 \%$ & $1 \mathrm{~dB}^{*}$ & $0.15 \lambda_{g c} \times 0.15 \lambda_{g c}{ }^{*}$ \\
{$[5]$} & 4 & 3 & $20 \%$ & - & $2 \mathrm{~dB}^{*}$ & $0.78 \lambda_{g c} \times 0.17 \lambda_{g c}{ }^{*}$ \\
{$[6]$} & 1.8 & 1 & $4 \%$ & $\dagger$ & $1 \mathrm{~dB}^{*}$ & $0.09 \lambda_{g c} \times 0.07 \lambda_{g c}{ }^{*}$ \\
{$[7]$} & 2 & 2 & $13 \%$ & $2.8 \%$ & $0.6 \mathrm{~dB}^{*}$ & $0.1 \lambda_{g c} \times 0.18 \lambda_{g c}$ \\
{$[8]$} & 5.4 & 4 & $35.5 \%$ & $11.2 \%$ & $2 \mathrm{~dB}^{*}$ & $0.49 \lambda_{g c} \times 0.28 \lambda_{g c}$ \\
{$[9]$} & 0.9 & 2 & $3.5 \%{ }^{*}$ & - & $1 \mathrm{~dB}^{*}$ & $0.27 \lambda_{g c} \times 0.3 \lambda_{g c}{ }^{*}$ \\
This & 1.9 & 2 & $9.3 \%$ & $3.7 \%$ & $1.3 \mathrm{~dB}$ & $0.31 \lambda_{g c} \times 0.19 \lambda_{g c}$ \\
work & & & & & \\
\hline \hline
\end{tabular}

† Single frequency point ${ }^{*}$ Estimated

for $-20-$ and $-30-\mathrm{dB}$ rejection levels are $9.3 \%$ and $3.7 \%$, respectively. The measured out-of-band insertion loss is $\mathrm{IL}<$ $1 \mathrm{~dB}$ in the lower passband and $\mathrm{IL}<1.3 \mathrm{~dB}$ in the upper passband up to $4 \mathrm{GHz}$.

Table II compares the fabricated filter performance to other fully uniplanar [2]-[4] and slotted- or patterned-ground, notuniplanar [5]-[9] BSFs reported in the literature. The proposed filter features a wider $-30-\mathrm{dB}$ rejection FBW with similar passband IL than other filters of a smaller size [4], [6], [7] and filters of the same order [4], [7]. Compared to the filters with wider -30-dB rejection FBW [2], [8], the proposed filter features a smaller size. Compared to [3], [5], and [9], the proposed filter is smaller and features a higher stopband rejection (greater than $30 \mathrm{~dB}$ ). It also features a smaller size (in terms of guided wavelength $\lambda_{\mathrm{gc}}$ ) than the other fully uniplanar filters [2], [3], and the not-uniplanar filters in [5], [8], and [9]. The estimated size of the proposed filter with a higher order would still be smaller $\left(0.46 \lambda_{\mathrm{gc}} \times 0.19 \lambda_{\mathrm{gc}}\right.$ for $n=3$ and $0.62 \lambda_{\mathrm{gc}} \times 0.19 \lambda_{\mathrm{gc}}$ for $\left.n=4\right)$ than the higher order filters in [2], [3], [5] $(n=3)$, and [8] $(n=4)$. The size reduction of the proposed filter is due to the slow-wave multimodal integration of inverters and resonators in the same CPW section.

\section{CONCLUSION}

A fully uniplanar BSF structure based on multimodal CPW resonators has been proposed. The multimodal theory has been used for the first time in the conception, modeling, and design of a BSF, resulting in a compact structure wherein resonators and inverters share the same circuit area. Moreover, it allows the use of CPW slow-wave periodic structures to achieve further compactness. The experimental results validate the filter concept and its theoretical analysis. The fabricated second-order filter features an FBW of $9.3 \%$ at $-20 \mathrm{~dB}$ and $3.7 \%$ at $-30 \mathrm{~dB}$, and an $\mathrm{IL}<1.3 \mathrm{~dB}$. The filter size is $0.31 \lambda_{\mathrm{gc}} \times 0.19 \lambda_{\mathrm{gc}}$.

\section{REFERENCES}

[1] S. Wallage, J. B. Tauritz, G. H. Tan, P. Hadley, and J. E. Mooij, "High T $C$ superconducting CPW bandstop filters for radio astronomy front ends," IEEE Trans. Appl. Supercond., vol. 7, no. 2, pp. 3489-3491, Jun. 1997.

[2] B. M. Karyamapudi and J.-S. Hong, "Coplanar waveguide periodic structures with resonant elements and their application in microwave filters," in IEEE MTT-S Int. Microw. Symp. Dig., Jun. 2003, pp. 1619-1622.

[3] J. Kim, C. S. Cho, and J. W. Lee, "CPW bandstop filter using slot-type SRRs," Electron. Lett., vol. 41, no. 24, pp. 1333-1334, Nov. 2005.

[4] A. Batmanov, E. Burte, R. Mikuta, A. Boutejdar, A. Omar, and A. Khaidurova, "Design of coplanar bandstop filter based on open-loopring resonator and DGS for WLAN and UWB applications," in Proc. Eur. Microw. Conf., 2012, pp. 743-746.

[5] D.-J. Woo, T.-K. Lee, J.-W. Lee, C.-S. Pyo, and W.-K. Choi, "Novel U-slot and V-slot DGSs for bandstop filter with improved Q factor," IEEE Trans. Microw. Theory Techn., vol. 54, no. 6, pp. 2840-2847, Jun. 2006.

[6] S. Y. Huang and Y. H. Lee, "A compact E-shaped patterned ground structure and its applications to tunable bandstop resonator," IEEE Trans. Microw. Theory Techn., vol. 57, no. 3, pp. 657-666, Mar. 2009.

[7] A. Ebrahimi, W. Withayachumnankul, S. F. Al-Sarawi, and D. Abbott, "Compact second-order bandstop filter based on dual-mode complementary split-ring resonator," IEEE Microw. Wireless Compon. Lett., vol. 26, no. 8, pp. 571-573, Aug. 2016.

[8] H. Chen, D. Jiang, and X. Chen, "Wideband bandstop filter using hybrid microstrip/CPW-DGS with via-hole connection," Electron. Lett., vol. 52, no. 17, pp. 1469-1470, Aug. 2016.

[9] Y.-H. Chun, R. Keller, J.-S. Hong, C. Fragkiadakis, R. V. Wright, and P. B. Kirby, "Lead-strontium-titanate varactor-tuned CPW bandstop filter on liquid crystal polymer substrates," in Proc. Eur. Microw. Conf., 2009, pp. 1373-1376.

[10] A. Contreras, M. Ribo, L. Pradell, and P. Blondy, "Uniplanar bandpass filters based on multimodal immitance inverters and end-coupled slotline resonators," IEEE Trans. Microw. Theory Techn., vol. 61, no. 1, pp. 77-88, Jan. 2013.

[11] M. Ribo and L. Pradell, "Circuit model for mode conversion in coplanar waveguide asymmetric shunt impedances," Electron. Lett., vol. 35, no. 9 , pp. 713-715, Apr. 1999.

[12] J.-S. G. Hong and M. J. Lancaster, "Highpass and bandstop filters," in Filters for RF/Microwave Applications. New York, NY, USA: Wiley, 2001, pp. 176-182. 